\title{
Variability of diffuse plaques and amyloid angiopathy in Parkinson's disease with mild cognitive impairment
}

\author{
Charles H. Adler • Thomas G. Beach
}

Received: 4 October 2010/Accepted: 5 October 2010/Published online: 13 October 2010

(C) Springer-Verlag 2010

We appreciate Professor Jellinger's correspondence [2] regarding our study [1] and his findings also showing heterogeneity of Lewy body and Alzheimer pathology in an additional eight cases of PD-MCI (four amnestic, four non-amnestic) [2]. Professor Jellinger questioned our lack of data on diffuse (amyloid) plaques and cerebral amyloid angiopathy (CAA), and so those data are presented.

As seen in the Table 1 , five cases had $3+$ diffuse amyloid plaque density in the cortex. Caudate-putamen amyloid plaque density ranged from 0 to $2+$. While we did not measure CAA in the meninges, we did in multiple cortical regions (frontal, temporal, parietal, and occipital) using a $0-3$ scale that was then totaled (maximum possible score $=12$ ). Four cases ( 3 amnestic, 1 non-amnestic) had CAA score of 0 , then one case each had scores of 1 (amnestic), 3 (non-amnestic), 4 (non-amnestic), and 5 (non-amnestic). All the cases with CAA score $\geq 1$ had a diffuse amyloid plaque density of $3+$.

We therefore agree with Professor Jellinger that further data are needed, with multivariate analysis, to determine if diffuse amyloid plaque load and CAA contribute to cognitive impairment in PD.

Table 1 Demographics and neuropathologic findings

\begin{tabular}{llllllllll}
\hline Age & Gender & $\begin{array}{l}\text { PD duration } \\
\text { (years) }\end{array}$ & $\begin{array}{l}\text { MCI } \\
\text { type }\end{array}$ & $\begin{array}{l}\text { Unified Lewy } \\
\text { body stage }\end{array}$ & $\begin{array}{l}\text { Neuritic } \\
\text { plaques }\end{array}$ & $\begin{array}{l}\text { Braak } \\
\text { AD stage }\end{array}$ & $\begin{array}{l}\text { Amyloid plaques, } \\
\text { cortex }\end{array}$ & $\begin{array}{l}\text { Amyloid plaques, } \\
\text { caudate putamen }\end{array}$ & $\begin{array}{l}\text { CAA total } \\
\text { score }\end{array}$ \\
\hline 74 & Male & 25 & N-A & Brainstem-Limbic & 0 & III & 0 & 0 & 0 \\
77 & Male & 14 & A & Brainstem & 0 & III & 0 & 0 & 0 \\
79 & Female & 17 & A & Neocortical & Moderate & IV & 3 & 0 & 0 \\
81 & Male & 11 & A & Brainstem & 0 & II & 0 & 2 & 1 \\
85 & Male & 13 & A & Brainstem-Limbic & Moderate & IV & 3 & 3 & 0 \\
88 & Female & 2 & N-A & Neocortical & Sparse & II & 3 & 3 \\
89 & Male & 7 & N-A & Brainstem-Limbic & Moderate & III & 3 & 3 \\
89 & Male & 2 & N-A & Brainstem & Moderate & III & 3 & 0 & 4 \\
\hline
\end{tabular}

$N$-A non-amnestic MCI, $A$ amnestic

C. H. Adler $(\bowtie)$

Department of Neurology, Mayo Clinic Arizona, 13400 E. Shea Boulevard, Scottsdale, AZ 85259, USA

e-mail: cadler@mayo.edu

T. G. Beach

Civin Laboratory for Neuropathology,

Banner Sun Health Research Institute,

Sun City, AZ, USA

\section{References}

1. Adler CH, Caviness JN, Sabbagh MN et al (2010) Heterogeneous neuropathological findings in Parkinson's disease with mild cognitive impairment. Acta Neuropathol. doi:10.1007/s00401010-0744-4

2. Jellinger K (2010) Neuropathology in Parkinson's disease with mild cognitive impairment. Acta Neuropathol. doi:10.1007/ s00401-010-0755-1 